\title{
Los búfalos en Argentina
}

\author{
Crudeli, G.A.'; Patiño, E.M. ${ }^{2}$; Maldonado, V.P. ${ }^{3}$; Konrad, J.L. ${ }^{3}$ \\ ${ }^{1}$ Cátedra Teriogenología; ${ }^{2}$ Cátedra Tecnología de los Alimentos, Carrera de Ciencias Veterinarias, UNCAUS. \\ ${ }^{3}$ Cátedra de Teriogenología, Facultad de Ciencias Veterinarias, UNNE. Comandante Fernández 755, \\ UNCAUS, Sáenz Peña, Chaco. Email: gacrudeli@hotmail.com
}

\begin{abstract}
Resumen
Crudeli, G.A.; Patiño, E.M.; Maldonado, V.P.; Konrad, J.L. Los búfalos en Argentina. Rev. Vet. 32: 2, 169-173, 2021. El búfalo fue introducido en Argentina a comienzo del siglo XX, mediante la importación de razas Mediterránea, Murrah y Jafarabadi. En la actualidad la población bubalina es de 147.785 cabezas distribuidas en 19 de las 23 provincias argentinas que componen el territorio nacional. El $88 \%$ de la población de búfalos se encuentra en el nordeste argentino, siendo las provincias de Corrientes y Formosa las que cuentan con las mayores densidades poblacionales. Existen actualmente 1193 productores de búfalos en todo el país. Las proyecciones de población bubalina para el año 2030 alcanzan la cifra de 430.000 cabezas.
\end{abstract}

Palabras clave: búfalo, población, productores, Argentina

\begin{abstract}
Crudeli, G.A.; Patiño, E.M.; Maldonado, V.P.; Konrad, J.L. The buffalos in Argentina. Rev. Vet. 32: 2, 169-173, 2021. The buffalo was introduced in Argentina at the beginning of the 20th century, through the importation of Mediterranean, Murrah and Jafarabadi breeds. At present the buffalo population is 147,785 heads distributed in 19 of the 23 Argentine provinces that make up the national territory. $88 \%$ of the buffalo population is found in the northeast of Argentina, being the provinces of Corrientes and Formosa the ones with the highest population densities. There are currently 1,193 buffalo producers across the country. The buffalo population projections for the year 2030 reach the figure of 432,262.
\end{abstract}

Key words: buffalo, population, producers, Argentina.

\section{LA HISTORIA}

Los primeros búfalos llegaron a la Argentina a principios del siglo XX, procedentes de Rumania y con destino a la Provincia de Entre Ríos, donde se intentó cruzarlos con bovinos para destinarlos a la producción de leche.

Obviamente fue un fracaso debido al intento de cruzar al búfalo con el ganado vacuno, sin considerar la incompatibilidad cromosómica debido que el búfalo tiene 50 cromosomas y el vacuno 60 . Luego de este fallido ensayo, los búfalos prácticamente desaparecieron, excepto algunos que fueron librados con destino a la caza mayor ${ }^{2,4}$.

Como no pudieron cruzarse, el fracaso hizo que se los fuese olvidando como animales útiles para la producción de leche y carne, destinándose para la caza. Así fue que a mediados de este siglo el búfalo que se encontraba en el país era destinado para el deporte conocido como de caza mayor.

En las provincias de La Pampa y Corrientes había establecimientos con rodeos destinados a este fin y ese concepto seguiría primando durante años. En la Provincia de Formosa, en 1973, se liberaron en el Centro Biológico de Pilagá III, un rodeo de 25 búfalos proce- dentes de Curuzú Cuatiá (Corrientes), para reproducirlos y criarlos en determinados lugares, como un atractivo para los aficionados a la caza mayor. El estudio que se hizo con ese rebaño durante los primeros años, demostró sus cualidades para la producción de leche y de carne en ambientes subtropicales.

Posteriormente el Ministerio de Asuntos Agropecuarios de la Provincia de Formosa decidió llevar un rodeo de 15 bubalinos a la Estación de Animales Silvestres Guaycolec, para que el público en general se fuera habituando a considerar al búfalo como un integrante más de la ganadería formoseña y no como una bestia salvaje.

Por otra parte, se distribuyeron pequeños planteles entre los ganaderos más evolucionados de los que integraban el Plan de Desarrollo Ganadero, con el objetivo de comenzar su explotación como animales productores de carne y leche. Estas medidas favorecieron la posterior radicación de establecimientos destinados a la explotación del búfalo en localidades como Riacho HéHé, Pirané, Espinillo y otras ${ }^{1}$.

En la década de los 70 nació el interés por los búfalos de parte de criadores que buscaban explotar campos bajos ubicados en la cuenca del río Paraná, hasta ese momento muy poco productivos con los vacunos. 
Concretamente, en 1976, en la Estancia Santa Rosa, ubicada en Esquina, Corrientes (propiedad de la familia Bencich), se comenzó a comprar lotes de destetes hembra de raza Mediterránea, procedentes de rodeos sin manejo (ariscos), hasta formar un rodeo de 1500 madres, importando después reproductores puros $\mathrm{Mu}$ rrah y Jafarabadi. Ese fue el verdadero comienzo de la explotación racional de búfalos en el país ${ }^{9}$

Recién a comienzos de la década de 1980 se reanudaron importaciones en pequeña escala, que fueron realizadas desde Italia, Brasil y Paraguay. Los ejemplares importados pertenecían a las razas Mediterráneo, Murrah y Jafarabadi ${ }^{2,8}$. En 1979 se importaron 100 animales de las razas Jafarabadi y Murrah; 40 de ellos fueron enviados a la Estancia Santa Rosa ubicada en la localidad de Esquina, Provincia de de Corrientes y los 60 restantes a las estancias La Florencia y La Alicia en la localidad de San Cristóbal, provincia de Santa Fé.

En 1983 se crea la Asociación Argentina de Criadores de Búfalos (AACB) bajo la presidencia de José Enrique Bencichyen. En 1985 se registraron los primeros planteles puros abriéndose el primer libro de registros genealógicos. En los años ' 90 se produjeron grandes importaciones, más de 5.000 vientres y reproductores seleccionados desde Brasil, lo cual consolidó la cría del búfalo en el país $5,7,9$.

Desde inicios de la década de los 90 la Facultad de Ciencias Veterinarias de la Universidad Nacional del Nordeste (UNNE) comenzó a desarrollar estudios sobre diversos aspectos del búfalo y consolidó un grupo de investigadores que gracias a su trabajo y publicaciones científicas tanto dentro como fuera de nuestro país, logró colocar a la UNNE como la más importante universidad argentina en el estudio del búfalo

En el año 2004 se funda en la ciudad de Corrientes, Argentina, la Asociación para la Producción y el Desarrollo del Búfalo en la Argentina (ABUAR), bajo la presidencia del Sr. H. F. Danuzzo. Esta asociación, resultado de una mancomunidad entre productores de búfalos de Corrientes y docentes e investigadores de la UNNE, permitió un mecanismo extraordinario para el estudio y difusión del búfalo en el país y en el exterior.

Gracias a ello, grupos de investigadores de la UNNE intensificaron los estudios sobre diversos aspectos del búfalo, que eran realizados en establecimientos bubalinos de productores de ABUAR y posteriormente los resultados de esas investigaciones eran transferidos a los productores para el mejoramiento de sus rodeos bubalinos. Gracias a esta conjunción se logró el reconocimiento mundial del búfalo en Argentina, debido fundamentalmente a la presencia permanente en congresos y simposios mundiales de búfalos, con presentación de trabajos científicos, conferencias, libros referidos a diversos aspectos del búfalo y dictados de cursos de grado y postgrado.

Fruto de este incansable trabajo mancomunado entre productores y técnicos, el mundo bubalino premió a la Argentina, primero eligiéndola para realizar el $I I$ Simposio de Búfalos de la Américas, que fue llevado a cabo con gran éxito en la ciudad de Corrientes en el año 2004 y posteriormente en el 2010 haciéndola sede del $X$ Congreso Mundial de Búfalos ${ }^{3}$, el cual se realizó con la presencia de gran cantidad de participantes de todo el mundo en las ciudades de Buenos Aires y Corrientes.

Finalmente, en el año 2015 el Congreso de la Nación Argentina promulga la Ley Nacional $\mathrm{N}^{\circ} 27.076$, denominada Programa Federal para el Fomento y Desarrollo de la Producción Bubalina. Cabe destacar que la mencionada ley fue sancionada por unanimidad parlamentaria y reglamentada en el año 2021 , lo cual permitirá contar con una herramienta formidable para la consolidación de la "bubalinocultura" en nuestro país.

\section{LA ACTUALIDAD}

En nuestro país existen en la actualidad, según datos oficiales, 147.785 cabezas de búfalos y un total de 1193 productores (Senasa, 2020). De acuerdo a los datos oficiales aportados por el Servicio Nacional de Sanidad Animal (SENASA), la población de búfalos en A rgentina en el año 2013, era de 87.711 cabezas ${ }^{6}$ (Tabla 1) y un total de 930 productores bubalinos (Senasa 2013) Esto significa un aumento del $68,4 \%$ en población de búfalos, desde diciembre/2013 hasta marzo/2020, en tanto, los nuevos productores bufaleros que se incorporaron a la actividad en este mismo lapso, fueron de 163 , llevando el total nacional de productores a la cantidad de 1193.

Es importante destacar que estos datos fueron de marzo de 2020, por lo cual a la fecha no están contemplados en este listado los animales recién nacidos o que nacieron hasta ahora, considerando en función de la estacionalidad que rige a esta especie.

Por lo tanto, si se considera que existían 89.632 vientres (búfalas y bubillas para servicio) y calculando un valor medio de preñez del $70 \%$, tendríamos más de 62.000 buceros nacidos, lo cual agregado al total registrado, sumarían más de 210.000 búfalos en nuestro país.

De las 23 provincias que componen el territorio nacional, existe población bubalina en 19 de ellas y solo en cuatro provincias del extremo sur argentino (Neuquén, Chubut, Santa Cruz y Tierra del Fuego), aún no se crían búfalos.

Tomando en consideración la cantidad de búfalos que poseen las provincias argentinas, pueden ser agrupados de la siguiente manera: más grandes de $19 \mathrm{mil}$ cabezas ( 3 provincias), medianas, entre mil y 6 mil ejemplares (7 provincias) y pequeñas con menos de mil cabezas (9 provincias), como puede visualizarse en la Tabla 1.

Las tres provincias con mayores poblaciones bubalinas, son Corrientes (60.671), Formosa (42.523) y Chaco (19.614). Teniendo en cuenta la división por regiones, la del Nordeste Argentino (NEA), integradas por Corrientes, Formosa, Chaco, Misiones y Entre Ríos, concentran el $88,89 \%$ de la población bubalina del país. La provincia de Corrientes es la que tiene el 
Tabla 1. Existencia de búfalos en Argentina, por provincias, categorías y productores.

\begin{tabular}{lccccccccccc}
\hline Provincia & $\mathrm{A}$ & $\mathrm{B}$ & $\mathrm{C}$ & $\mathrm{D}$ & $\mathrm{E}$ & $\mathrm{F}$ & $\mathrm{G}$ & $\mathrm{H}$ & $\mathrm{I}$ & $\mathrm{T}$ & $\mathrm{P}$ \\
\hline Corrientes & 2 & 4.467 & 2.644 & 7.139 & 6.177 & 9 & 1.897 & 28.772 & 9.564 & 60.671 & 287 \\
Formosa & 2 & 2.764 & 1.890 & 4.746 & 4.378 & 166 & 913 & 21.207 & 6.457 & 42.523 & 156 \\
Chaco & 7 & 2.207 & 1.761 & 1.773 & 1.625 & 80 & 447 & 8.470 & 3.244 & 19.614 & 173 \\
Entre Rios & 1 & 493 & 497 & 800 & 681 & 22 & 146 & 1.610 & 1.044 & 5.294 & 100 \\
Santa Fe & 0 & 1.591 & 341 & 379 & 480 & 52 & 55 & 1.568 & 372 & 4.838 & 54 \\
Bs.Aires & 8 & 393 & 296 & 590 & 671 & 80 & 101 & 1.069 & 578 & 3.786 & 90 \\
Misiones & 9 & 168 & 104 & 476 & 546 & 11 & 81 & 1.114 & 768 & 3.277 & 81 \\
S. Estero & 9 & 419 & 153 & 256 & 268 & 136 & 58 & 1.245 & 292 & 2.836 & 51 \\
Córdoba & 0 & 209 & 66 & 165 & 238 & 51 & 82 & 879 & 148 & 1.838 & 53 \\
La Pama & 0 & 45 & 102 & 342 & 332 & 54 & 234 & 584 & 143 & 1.836 & 32 \\
San Luis & 0 & 0 & 0 & 28 & 38 & 43 & 65 & 159 & 96 & 429 & 18 \\
Salta & 0 & 22 & 32 & 24 & 23 & 69 & 104 & 31 & 20 & 325 & 32 \\
Mendoza & 0 & 5 & 0 & 23 & 41 & 10 & 19 & 35 & 56 & 189 & 18 \\
Tucumán & 9 & 27 & 33 & 17 & 5 & 2 & 3 & 18 & 38 & 152 & 22 \\
Catamarca & 26 & 0 & 0 & 0 & 0 & 13 & 0 & 10 & 0 & 49 & 4 \\
Jujuy & 5 & 15 & 3 & 0 & 1 & 1 & 12 & 7 & 0 & 44 & 14 \\
San Juan & 0 & 0 & 5 & 7 & 1 & 4 & 6 & 0 & 20 & 43 & 4 \\
Rio Negro & 0 & 0 & 2 & 4 & 2 & 0 & 8 & 14 & 0 & 30 & 2 \\
La Rioja & 0 & 0 & 0 & 1 & 0 & 10 & 0 & 0 & 0 & 11 & 2 \\
Total & 78 & 12.825 & 7.929 & 16.770 & 15.507 & 813 & 4.231 & 66.792 & 22.840 & 147.785 & 1.193 \\
\hline
\end{tabular}

Fuente: SENASA (2020). Categorías: A: buey (buey búfalo), B: novillito (bubillito), C: novillo (bubillo), D: ternera (bucerra), E: ternero (bucerro), F: torito (butorito), G: toro (búfalo), H: vaca (búfala), I: vaquillona (bubilla), T: total, P: productores.

mayor número de cabezas de búfalo y cantidad de productores bufaleros.

Al evaluar el incremento individual de cada provincia grande, notamos que en el año 2013 Corrientes poseía 23.397 ejemplares. En la actualidad posee 60.671 ejemplares, lo que hace un incremento total del orden de $160 \%$, con respecto al año 2013 .

En esta misma línea, la Provincia del Chaco sumó un $133 \%$ de aumento en el mismo período, en tanto que en Formosa el aumento fue sólo del $26 \%$, en dicho lapso. Una explicación a este bajo incremento podría ser que esta provincia, que posee establecimientos con un gran número de cabezas, haya vendido muchos vientres hacia otras provincias, siendo Chaco y Corrientes las principales beneficiarias.

Cuando nos abocamos a la situación de las llamadas medianas, encontramos algunos datos interesantes como ser, que la provincia de Buenos Aires, es la única que disminuyó la cantidad de cabezas a la mitad. Entre Ríos tuvo un leve aumento de su stock bubalino, en tanto Misiones, Santiago del Estero, Córdoba y La Pampa, incrementaron notablemente sus rodeos, siendo Santiago del Estero, la provincia líder en este aspecto, ya que casi cuadruplicó su población.

Un hecho para destacar es La Pampa, que posee casi 300 machos, para una población de hembras de 650 , es decir un toro cada 2 búfalas, lo que habla que aquí influye mucho el tipo de actividad a la que son sometidos, en este caso a cotos de caza.

Con relación a lo antes mencionado, cabe destacar que existen en el país cotos de caza que ofrecen búfalos de agua (Bubalus bubalis) para actividades cinegéticas, en las provincias de La Pampa, San Luis, Rio
Negro, Buenos Aires, Entre Ríos, Córdoba, Neuquén, Formosa y Corrientes. Los búfalos destinados a esta actividad son machos adultos de grandes cuernos que ya no operan como reproductores en los establecimientos bubalinos.

Debemos mencionar un hecho muy auspicioso para la ganadería bubalina del país, como lo es la creación del Centro Integral de Inseminación Artificial Bubalino (CIIAB), aprobado por Senasa y ubicado en Paraje Florentín, en cercanías de General Paz, Corrientes, el cual se concretó hace un año y que cuenta con semen de reproductores de la raza Murrah y Mediterránea, de excelente calidad, lo que contribuirá sin dudas a acelerar el crecimiento genético del búfalo en Argentina.

Es importante destacar el destino de la producción de búfalos en nuestro país. A diferencia de lo que acontece en todos los países americanos y de los demás continentes donde se crían búfalos cuyo destino principal es la producción de leche, en Argentina el destino es la producción de carne. Pero debe hacerse una importante aclaración: a pesar de que en el país se crían como búfalos y se los faena como búfalos, la inmensa mayoría de esa producción se la comercializa como vacunos.

En lo que respecta a la producción de leche, la misma sigue siendo de muy escasa importancia. Sin embargo, existen importantes establecimientos productores de leche y elaboradores de excelentes productos derivados en provincias como Buenos Aires donde debe mencionarse a las empresas Arribata y La Delfina. En la provincia de Santiago del Estero, se conformó hace un año "La Bufalita", que vende sus productos en la ciudad de Córdoba. 
En tanto, en provincias como Formosa, Misiones y Corrientes, existen productores que elaboran productos artesanales con leche bubalina, como quesos mozzarella y de tipo criollo, además de dulce de leche. En las Facultades de Ciencias Veterinarias de la Universidad Nacional del Nordeste (UNNE) en la provincia de Corrientes y en la Universidad Nacional del Litoral (UNL) en Esperanza, provincia de Santa Fe, existen mini plantas lácteas que elaboran productos derivados de la leche bubalina que tienen una finalidad didáctica para alumnos y pequeños productores.

\section{EL FUTURO}

El escenario futuro resulta promisorio. Si consideramos que según datos de SENASA el crecimiento de la población bubalina ronda el $9 \%$, la proyección para el año 2030 nos da una cifra de alrededor de 430.000 bubalinos. Desde la producción primaria se observa no sólo una intensificación de los sistemas en busca de mejores índices productivos, sino también un aumento de las inversiones en nuevas actividades complementarias que contribuyan al mix de ingresos de los establecimientos.

Teniendo en cuenta que la seguridad alimentaria será una prioridad en el futuro: en los próximos 30 años, las necesidades mundiales de alimentos serán más del doble como consecuencia del crecimiento de la población, el desarrollo económico, los cambios radicales en los patrones de consumo y el incremento del consumo de productos pecuarios.

La Argentina presenta regiones inexplotadas o explotadas ineficientemente desde el punto de vista pecuario, debido a la falta de adaptación del ganado vacuno a la misma, como son los sectores bajos e inundables que totalizan unos ocho millones de hectáreas. Tal superficie $\left(80.000 \mathrm{~km}^{2}\right.$ ) comprende $15.000 \mathrm{~km}^{2}$ de bajos sub-meridionales santafesinos; $10.000 \mathrm{~km}^{2}$ de costa de los ríos Paraná y Paraguay en Santa Fé, Chaco y Formosa; $20.000 \mathrm{~km}^{2}$ del pre-delta entrerriano y delta bonaerense; $15.000 \mathrm{~km}^{2}$ de esteros correntinos y 20.000 $\mathrm{km}^{2}$ de la cuenca del Río Salado en Buenos Aires.

Esta colosal superficie, es apta para soportar la carga de un búfalo cada dos hectáreas, lo que permitiría una población de 4 millones de búfalos, que no competirían con el ganado tradicional, el cual tiene baja eficiencia productiva en estas zonas "marginales".

La producción de búfalos en el país y principalmente en la región NEA, presenta condiciones y ventajas competitivas para conformar un entramado o cluster productivo exitoso, ya que cuenta en su territorio con el ecosistema adecuado, también con el socio-sistema, es decir los productores dedicados a la actividad bubalina, y como tercer componente, existen instituciones de generación de tecnología como facultades de veterinaria y agencias del INTA, muchas de las cuales vienen dedicándose desde tiempo atrás a la investigación de temáticas referidas a la producción e innovación tecnológica para la producción bubalina, articulando y coordinando acciones con organismos provinciales y nacionales.

Varios factores dan indicio que existe un horizonte de mayor certeza y optimismo en el futuro de la actividad El reconocimiento desde el ámbito oficial, de sector estratégico por sus posibilidades de desarrollo y crecimiento, con la reglamentación y puesta en funcionamiento de la Ley No 27.076 Programa Federal para el Fomento y Desarrollo de la Producción Bubalina.

Las perspectivas de crecimiento de la demanda de carne: en el mercado interno como sustituto natural de la carne bovina, en un escenario probable de crecientes volúmenes de exportación de esta carne al mercado externo. Dentro de este análisis la carne de búfalo corre con ventajas comparativas en seguridad, condiciones de producción en relación a bienestar animal y calidad de los alimentos ya que estos serán definitorios en una cada vez más integrada cadena alimentaria.

Los alimentos sanos y la ingesta de carne en relación con la salud humana, las enfermedades crónicas, el envejecimiento y su impacto en la vida, serán factores claves

en la industria ganadera en un futuro. Si a esto agregamos promover al mejoramiento productivo de los pequeños productores facilitando el acceso a la capacitación y a la incorporación de tecnología, Argentina cuenta con ventajas objetivas para llegar a ser un gran productor de búfalos.

$¿$ Resta plantear una agenda futura pensando en lo que vendrá ?

El cambio climático, el aumento de las sequías o inundaciones en los países, la crisis económica mundial, los conflictos de la pandemia por COVID-19 y otros factores desconocidos, provocarán un incremento de la volatilidad de los precios de los alimentos y será un verdadero reto que deberá priorizar una visión compartida para la producción de búfalos en el país y en la región, posicionando a los actores como un espacio propenso a la competitividad y a la innovación.

\section{REFERENCIAS}

1. Carrazoni, J.A. 1993. El búfalo y su importancia para el futuro. Articulo (14 pag.) Universidad Nacional de La Plata (UNLP). Argentina.http://sedici.unlp.edu.ar/ bitstream/handle/10915/30210/Documento_completo. $p d f$ ? sequence $=1$.

2. Carrazoni, JA. 1998. El búfalo en el mundo y en nuestro pais. Vet Arg 79: 2-7.

3. Congreso de la Nación Argentina. 2015. Ley 27076. Programa federal para el fomento y desarrollo de la producción bubalina. htips:/www.argentina.gob.ar/ normativa/ nacional/ley-27076-240647.

4. Incahusti D, Tagle ZC. 1980. Bovinotecnia: Capitulo 9: Origen de los bovinos domésticos y bovideos, El Ateneo, Buenos Aires, p. 88-89.

5. Mastropolo R, Cravero J, Zava M. 1980. El búfalo. Rev Crea 85: 67-70. 
6. Servicio Nacional de Sanidad Animal (SENASA). 20132020. http:// www. senasa.gov.ar.

7. Smaldone O. 1995. Cría de búfalos. Un establecimiento modelo. Rev Acaecer 227: 32-34.

8. Zava MA. 2011. El búfalo doméstico, Cap. 14. Editora Orientación Gráfica, Buenos Aires, p. 521-596.
9. Zava MA. 2017.40 años del búfalo en Argentina. CES Virtual. 7 pag. https: //virtual.ces.edu.co/pluginfile.php/241210/ mod label/intro $1 . \% 2040 \% 20$ a $\%$ C3\%B1os\%20de $\% 20$ B\%C3\%BAfalo\%20en\%20Argentina_Marco\%20Zava.pdf 